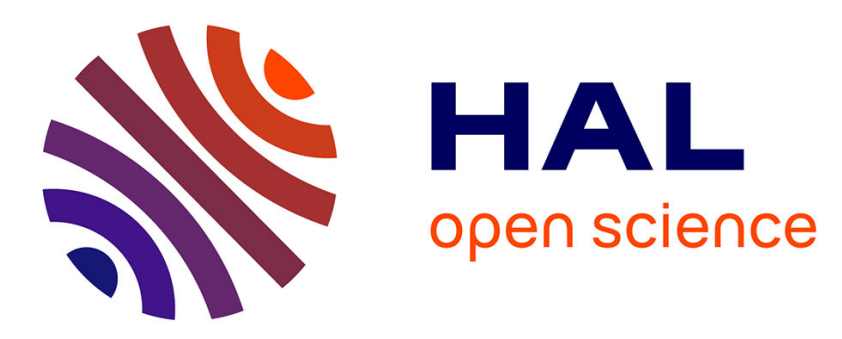

\title{
An investigation into the fracture behaviour of honeycombs with density gradients
}

Riccardo Manno, Josiane Nguejio, Fabrice Barbe, Ivano Benedetti

\section{To cite this version:}

Riccardo Manno, Josiane Nguejio, Fabrice Barbe, Ivano Benedetti. An investigation into the fracture behaviour of honeycombs with density gradients. International Conference on Fracture and Damage Mechanics, Sep 2020, Mallorca (On Line), Spain. pp.020015, 10.1063/5.0033972 . hal-02971959

\section{HAL Id: hal-02971959 \\ https://hal.science/hal-02971959}

Submitted on 20 Oct 2020

HAL is a multi-disciplinary open access archive for the deposit and dissemination of scientific research documents, whether they are published or not. The documents may come from teaching and research institutions in France or abroad, or from public or private research centers.
L'archive ouverte pluridisciplinaire HAL, est destinée au dépôt et à la diffusion de documents scientifiques de niveau recherche, publiés ou non, émanant des établissements d'enseignement et de recherche français ou étrangers, des laboratoires publics ou privés. 


\title{
An investigation into the fracture behaviour of honeycombs with density gradients
}

\author{
Riccardo Manno, 1, a) Josiane Nguejio, ${ }^{2,(b)}$ Fabrice Barbe,, , c) and \\ Ivano Benedetti ${ }^{3, d)}$ \\ ${ }^{1)}$ Bristol Composite Institute (ACCIS), Department of Aerospace Engineering, Queen's Building, University Walk, Bristol, BS8 \\ $1 T R$, United Kingdom \\ 2) INSA Rouen, UNIROUEN, Groupe de Physique des Matériaux, Normandie Univ, UMR CNRS 6634, Rouen, F-76000, France \\ ${ }^{3)}$ Department of Engineering, University of Palermo, Viale delle Scienze, Edificio 8, 90128, Palermo, Italy \\ a) Electronic mail: r.manno@bristol.ac.uk

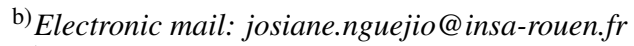

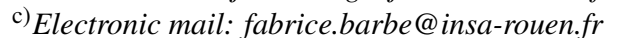 \\ d)Corresponding author: ivano.benedetti@unipa.it
}

\begin{abstract}
In this study we perform an experimental and computational investigation about the fracture behaviour of polymer honeycombs presenting gradients in terms of lattice density. Such lattice relative density variations are introduced with the aim of mimicking the micro-morphology encountered in some natural materials, such as several kinds of woods, which seems related to the ability of the corresponding macro-material to delay the propagation of fracture under certain conditions. Starting from the conclusions of previous computational analyses, we perform a few experimental tensile tests on ABS model honeycombs obtained by additive manufacturing, with the aim of getting insights into their fracture behaviour and assessing the effect of the density gradients on the failure process, with respect to the behaviour observed in baseline homogeneous lattices. Following the performed tests, novel finite element analyses are performed, to help explain the observed failure processes and as preliminary calibration for further investigations addressed at maximising the lattice fracture toughness under tensile loading. With the emerging of more reliable and affordable additive manufacturing technologies, the present study contributes to exploring the possibility of modifying the effective properties of honeycombs and lattice materials through sensible small modifications of the micro-morphology.
\end{abstract}

\section{To cite this submitted version:}

R. Manno, J. Nguejio, F. Barbe, I. Benedetti (2020).

An investigation into the fracture behaviour of honeycombs with density gradients.

Submitted to AIP. Proceedings of International Conference on Fracture and Damage Mechanics, Mallorca, Spain. 14-17th September, 2020.

\section{INTRODUCTION}

The development and employment of materials with suitable balances of sometimes contrasting properties, such as light weight and strength, stiffness and toughness, has always been inherently connected with the advancement of engineering and technology [1]. In the last few decades however, a special sensitivity has arisen in some sectors, e.g. automotive and aerospace, towards the development of materials able to deliver their structural performance, possibly together with some other function, in a multi-functional perspective, at the lowest possible wight. In such fields, lower weight directly implies reduced fuel consumption at the same payload level, with obvious perceived benefits in terms of operational capability and environmental impact.

In the sketched background, composite materials provide a remarkable example, confirmed by an impressive rise in the number of academic and industrial studies and subsequent applications. For structural and multifunctional lightweight applications, increasing interest is also being attracted by the development or enhancement of other kinds of materials: metallic foams [2] and micro-lattices [3] offer an array of interesting properties such as low density, relatively high stiffness, high energy absorption, mechanical damping, acoustic and thermal absorption, tunable porosity. In 2015, the announcement of the creation of the "world's lightest metal", in fact a micro-lattice, by Boeing in collaboration with the HLR Laboratories made headlines [4], confirming the growing attention in the field. Despite their appealing features, a wider deployment of such materials in structural applications has been so far hindered by a certain scatter of their mechanical properties [5, 6], often due to a lack of control in the manufacturing processes, which poses a certain difficulty in the certification processes required for critical operations. 
However, the recent fast developments in Additive Manufacturing (AM) technologies, with growing levels of quality control, have shown the possibility of manufacturing materials with multifunctional properties at a remarkable level of scale resolution [7]. The use of such technologies, combined with structural optimisation tools [8], offers unprecedented opportunities to fill voids in the material design space, paving the way towards the developments of architectured materials [9] delivering sought performances as a consequence of their micro-architecture [7].

While the use of such novel technologies may offer new opportunities for materials manufacturing, the materials design space to be explored, before delivering materials with the desired properties, might be too large and costly, even with the employment of optimisation tools. In this respect, Nature is often regarded as an invaluable source of inspiration, providing countless examples of materials able to deliver outstanding properties, thanks to an optimised hierarchical multi-scale arrangement of their elementary constituents [10], thus suggesting the possibility of mimicking such strategies in a bio-inspired materials design paradigm.

In this work, we present some preliminary results about the fracture performance of honeycombs exhibiting lattice density variations, similar to those observed in several kinds of woods and related to the ability of slowing crack propagation. The study extends the work performed in Refs. [11, 12] with some experimental results about the tensile fracture behaviour of ABS specimens, obtained by fused deposition modelling, and their finite element interpretation.

\section{TENSILE FAILURE EXPERIMENTAL TESTS}

The investigation presented here moves from the computational analysis carried out in a previous study. In particular, an experimental campaign on ABS lattices is started with the aim of assessing the observations put forward in Ref. [12] and, ultimately, evaluating the effects of lattice density gradients on the tensile failure behaviour of this kind of materials/structures.

Following preliminary computational indications, lattices identified by the ratio $R=0.6$ are considered, as they should present the highest increment of fracture energy with respect to the reference homogeneous lattices. It is reminded that, in a lattice with alternating bands of different relative density, if $l_{c}$ is the width of the lower density bands and $l_{f}$ the width of higher density bands, the ratio $R$ is defined by

$$
R=\frac{l_{f}}{l_{c}+l_{f}} .
$$

From the geometrical point of view, besides selecting the value of $R$, the test specimens have been designed considering the constraints related with the printing technology, the loading device and the tensile mechanical conditions. In particular: $a$ ) the overall specimens dimensions, as well as the characteristic metrics of the lattice, i.e. the size of the cells and walls, the number of cells, the number of bands, have been set as a trade-off between the building plate dimensions and the printing resolution; $b$ ) in the printed specimens the lattice region is attached to two non-lattice full parts - see Fig. [1 - designed and printed so to provide suitable grips to the loading machine and to transmit quasi-uniform vertical displacements to the lattice upper and lower ends upon loading; $c$ ) the notch length has been determined, from a preliminary finite element analysis, as the minimal length ensuring that the stress in a vertical cell wall reaches a maximum value at the notch tip, thus avoiding crack initiation elsewhere in the lattice.

The geometry and loading of the specimens is further described in Fig. 2. Given the geometry and the position of the grips, the un-notched specimen deforms as shown in Fig. 2 a, which results from the finite element simulation of the tensile test, assuming isotropic linear elastic properties for the material constituting the whole specimen. In this situation, due to the imperfect transmission of the displacement boundary conditions, higher values of axial stress are established in the central region of the specimen, as shown by the contour plot of $\sigma_{y y}$ in Fig. 2a. On the other hand, Fig. $2 \mathrm{p}$ shows the stress distribution in a notched specimen, illustrating how the notch length has been selected, so to ensure that fracture initiates from the notch tip, due to the higher value of $\sigma_{y y}$ in vertical walls located in its proximity.

From the manufacturing point of view, the specimens have been realised by ABS Filament Fusion Fabrication on a 3DGence Industry F340 3D printer. The infill density and the layer height have been set at $100 \%$ and $50 \mu \mathrm{m}$ respectively.

To characterise the tensile properties of 3D-printed ABS, standard tensile test specimens have been printed in the same conditions as the lattice specimens and according to different orientations on the building plate, i.e. at $0^{\circ}, 45^{\circ}$, $90^{\circ}$ and $135^{\circ}$ with respect to the front side of the plate. Following the procedure established in Ref. [13] about the characterisation of 3D-printed-ABS properties, the tensile tests have been performed after 2-week storage in cool and dry atmosphere, to allow the relaxation of the material after processing, imposing a displacement rate of $1 \mathrm{~mm} / \mathrm{min}$. The tensile curves and the corresponding mean tensile properties are presented in Fig. 3 . As classically observed 

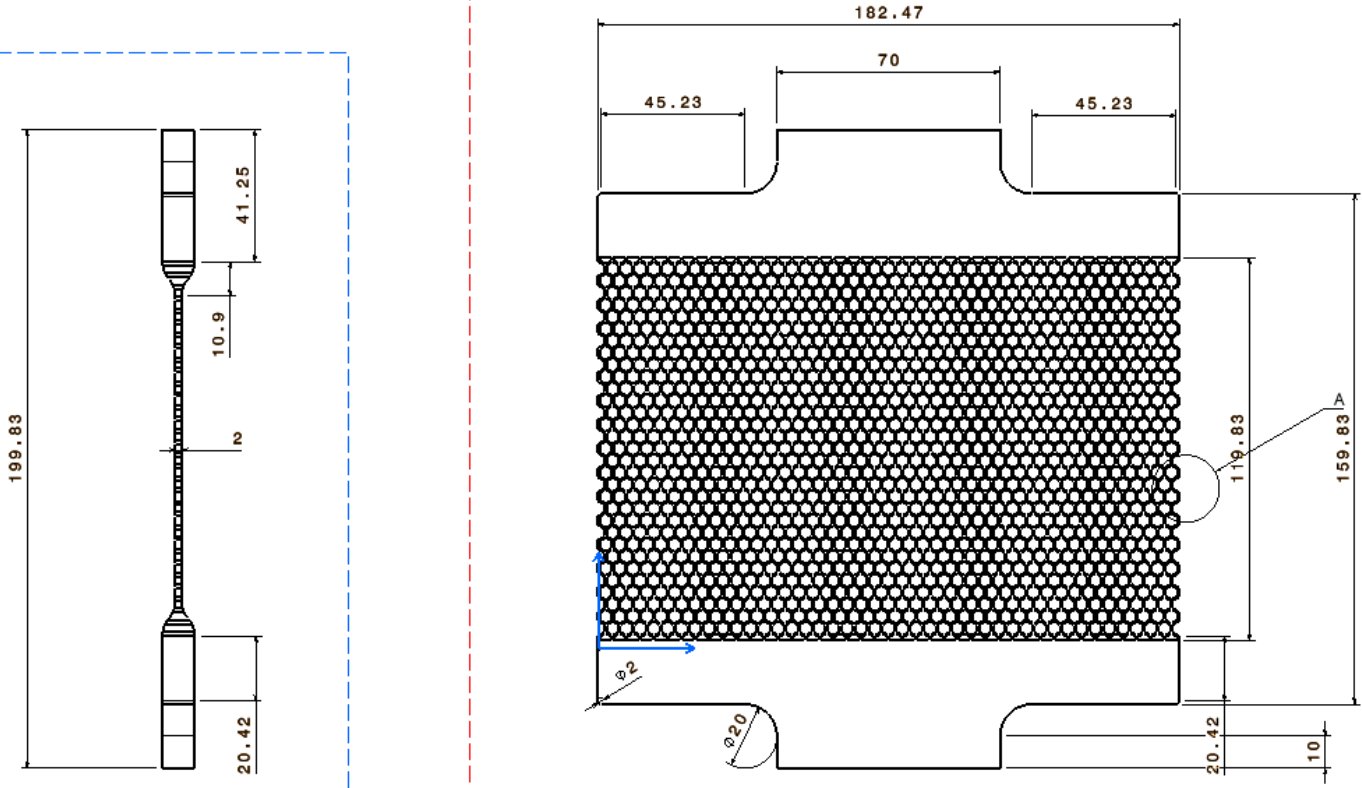

FIGURE 1: Example of specimen with density gradients for experimental analyses. The cell walls thickness is $t=0.54 \mathrm{~mm}$. Left: side view. Right: front view.

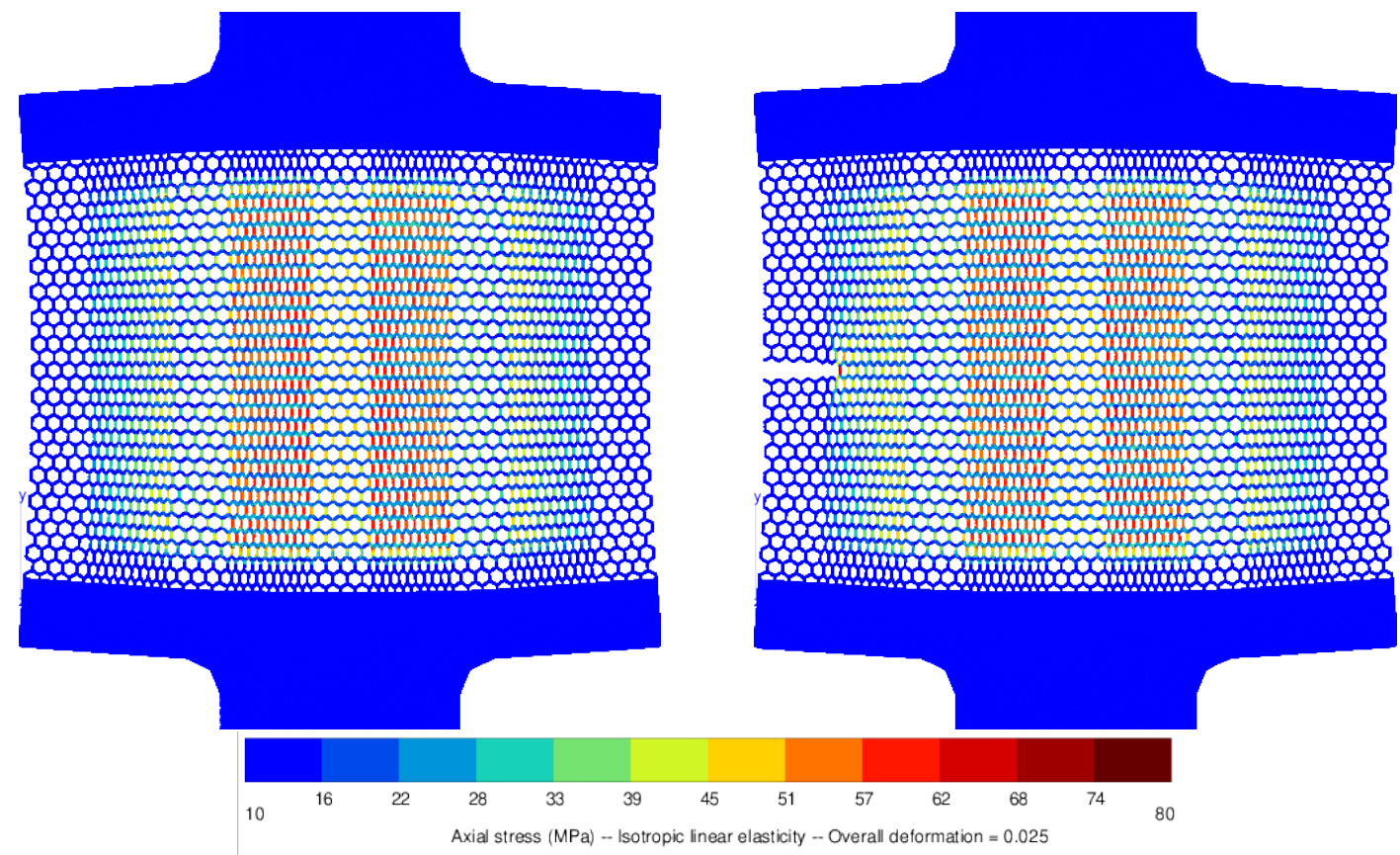

FIGURE 2: Spatial distribution of the axial (vertical) stress $\sigma_{y y}$ in the loaded non-homogeneous lattice as provided by FE simulation of the tensile test with assumed material isotropic linear elastic constitutive behaviour. Left: un-notched specimen. Right: notched specimen. 


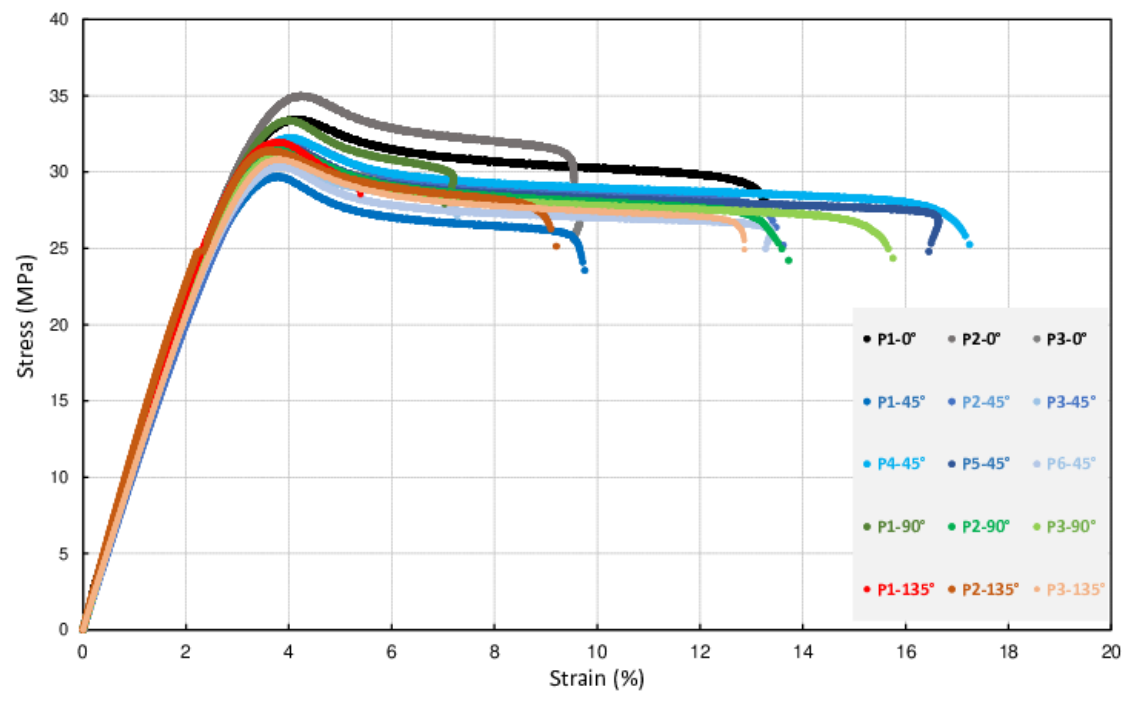

\begin{tabular}{c|c|c|c}
$\begin{array}{c}\text { Young modulus } \\
{[\mathrm{MPa}]}\end{array}$ & $\begin{array}{c}\text { Poisson ratio } \\
v\end{array}$ & $\begin{array}{c}\text { Ultimate tensile stress } \\
{[\mathrm{MPa}]}\end{array}$ & $\begin{array}{c}\text { Elongation to fracture } \\
{[\%]}\end{array}$ \\
\hline 1100 & 0.35 & 32 & 11.3
\end{tabular}

FIGURE 3: Top: tensile stress-strain curves of different ABS samples printed with different orientations on the building plate. Bottom: material parameters extracted from the tensile tests.

concerning ABS, the stress response increases linearly with strain up to a maximum stress of about $30 \mathrm{MPa}$ and then reaches a plateau eventually leading to fracture. As compared to the ultimate tensile stress, the elongation to fracture is highly dispersed, as also noted in Ref. [13], and no significant effect of the building orientation could be observed, which suggests that the mechanical properties may reasonably be considered as isotropic at a macro-scale. Such isotropy can be explained by the fact that the filament deposition orientation is switched by an angle of $45^{\circ}$ from layer to layer; this is programmed by the slicer software in order to favour isotropy - such as in a fibre-matrix laminates and also to reduce the amount of porosity.

Summarising, lattice specimens with a $25 \mathrm{~mm}$-long horizontal notch have been subjected to displacement controlled loading at the rate of $1 \mathrm{~mm} / \mathrm{min}$. Currently, 3 tests have been performed on notched specimens exhibiting density gradients and all the tests led to cracks propagating from the notch to the opposite side of the specimen, as shown in Fig. 4. The obtained experimental force-displacement curves are reported in Figs. (6.77) where they are also compared with the results of the finite element analysis discussed in the next section. It is observed that such curves show a good level of reproducibility, which is the necessary condition to exclude the possibility that printing defaults, necessarily present in the printed specimens, be the major structural characteristic governing the fracture propagation. In few of the manufactured specimens, undetectable printing defects initiated fracture events in regions far from the notch tip; such specimens have been thus excluded from the analyses.

A certain number of other tests, originally planned as part of this study, could not be performed as a consequence of the lockdown enforced throughout Europe to contrast the current outbreak of the CoViD-19 pandemic [14]. In particular, we could not perform tests on reference baseline homogenous lattices; for such a reason, in this study, only the response of honeycombs with density gradients has been experimentally investigated, while the response of homogeneous lattices has been estimated both analytically and numerically, without experimental validation. The presented results should then be intended as preliminary; however, the study, up to the forced interruption, has allowed the establishment a sound workflow for specimen manufacturing, testing and simulation, which will be useful in the planned continuation. 

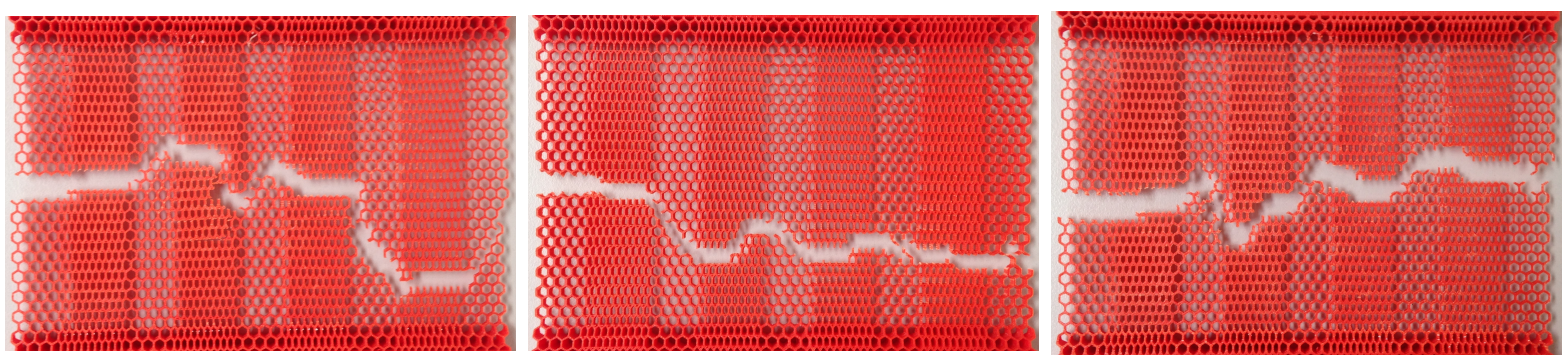

FIGURE 4: Post-failure configuration of ABS lattice specimens under progressive tensile loading; crack propagation initiated from the notch on the left hand side.

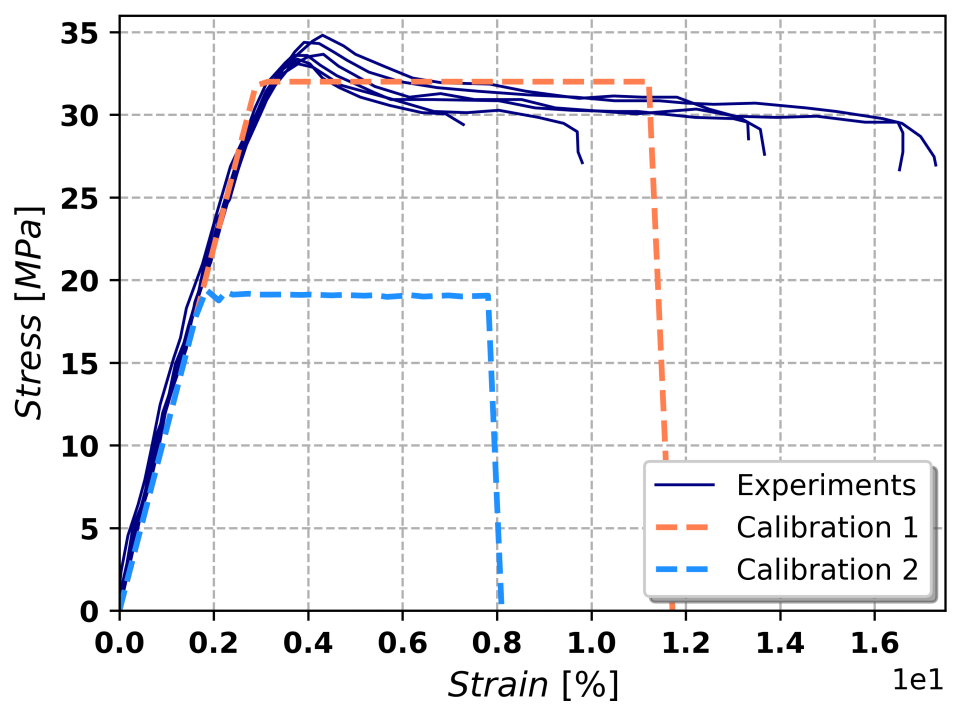

FIGURE 5: Material constitutive model calibrated against the experimental tensile tests performed on printed bulk ABS.

\section{COMPUTATIONAL FAILURE ANALYSIS}

The finite element analysis has been performed in ABAQUS/Explicit, identified as the suitable tool to investigate the highly non-linear processes of crack initiation, propagation and failure in polymeric lattices.

The geometry of the investigated honeycombs, their mesh and the sets of appropriate boundary conditions have been generated using a dedicated Python pre-processor developed in-house. In particular, for reducing computational costs, only the geometry of the cellular portion of the specimens has been generated, while the full parts manufactured for easing the specimens clamping have been neglected. Hence, to mimic the test loading conditions, the boundary conditions have been applied enforcing a parabolic displacement profile at the edges of the virtual specimen. The geometry has been meshed using quad shell element with plane strain formulation (CPE4R) and element deletion has been enabled. The constitutive behaviour of the parent material has been initially calibrated using the experimental tests on the printed bulk ABS reported in Fig. 3. Specifically, the constitutive behaviour has been approximated in this stage as shown in Fig. 5. i.e. as an ideal material exhibiting a short flat plateau, after an initially elastic response, followed by a narrow softening segment.

The set of numerical results obtained from the modelling strategy described above are are reported in Figure 6 , where they are compared with the experimental results available for the the tested honeycombs with density gradients and with the solution obtained from the analytical estimation of the stiffness of a honeycomb with homogeneous cellular density [1]. It is observed that, while the slope of the initial linear elastic response is satisfactorily captured by 


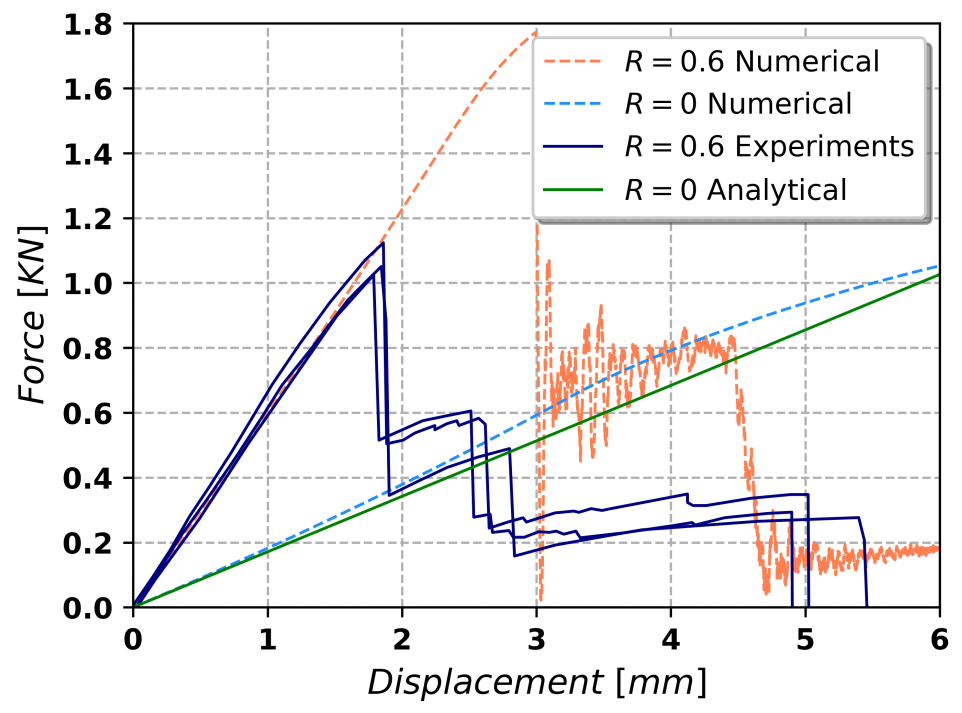

FIGURE 6: Force VS displacement curves for honeycombs with $R=0$ and $R=0.6$.

the numerical model, both the peak force and the failure strain are overestimated. The reasons behind such discrepancies might be related to a higher number of material defects in the printed honeycomb, with respect to the number of defects in the standard tensile test specimens used for inferring the material properties, and/or to the possible presence of a scale effect affecting the fracture behaviour of structural elements at different scales.

To get further insights into the influence of the parent material on the response of the honeycomb, a sensitivity analysis on the effect of the material constitutive properties is performed. Fig. 7 shows the load-displacement curves obtained when the parent material is modelled starting from the reduced constitutive curve plotted in Fig. 5. With such an assumption about the parent material, the slope of the elastic segment, the peak force and the strain at the onset of cracking are captured, which seems to confirm that the actual parent material is weaker that that inferred by the standard tensile tests. Furthermore, the crack path in the virtual specimen, reported in Fig. 8, is qualitatively compatible with what observed in the performed tests, see Fig. 4

\section{CONCLUSION}

In this study, a sound workflow for the manufacturing, experimental tensile testing and computational analysis of honeycombs exhibiting density gradients has been established and some preliminary experimental and computational results about the tensile fracture behaviour of such cellular materials have been presented. Good experimental reproducibility of the fracture behaviour across the specimens has been observed: the curves are characterised by four main stages occurring at quite similar imposed displacements and leading to similar force evolution. Some discrepancy between the behaviour of the tested specimens and the simulated response has been identified, both in terms of peak load and failure strain, when the constitutive behaviour of the parent material is inferred starting from printed standard tensile specimens. On the basis of some sensitivity analysis on the influence of the parent material properties on the honeycomb failure, it has been hypothesised that such discrepancies may be related to the influence of inevitable manufacturing defects and/or the presence of a material scale effect. However, such aspects will be further investigated in the continuation of the present research.

\section{ACKNOWLEDGMENTS}

Ivano Benedetti acknowledges the support of INSA Rouen Normandie, where he has been invited as Foreign Visiting Professor between November and December 2019. 


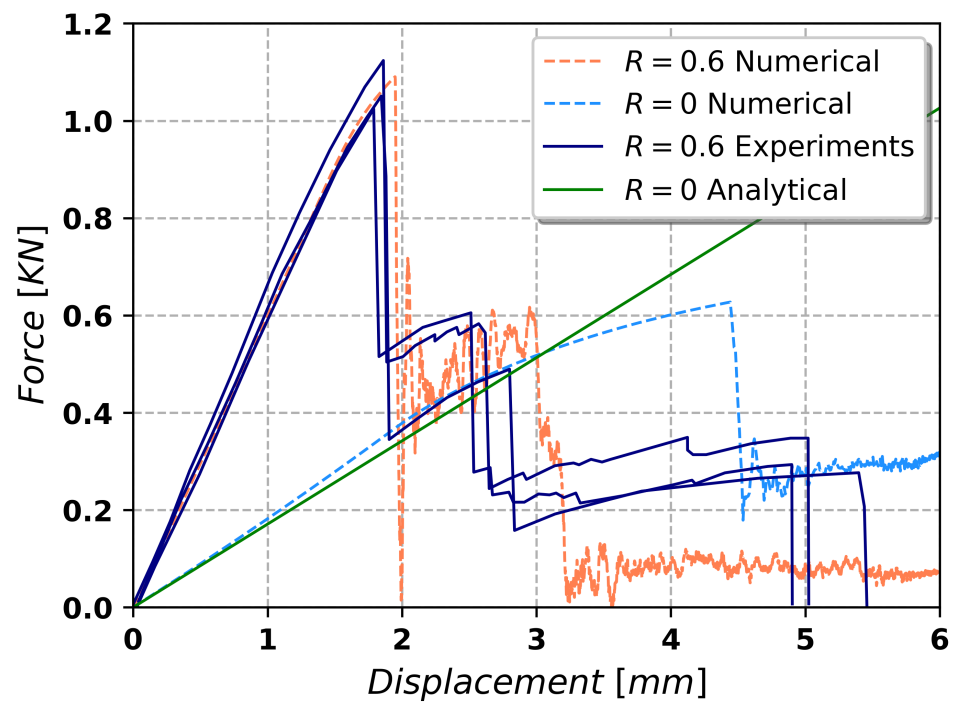

FIGURE 7: Force VS displacement curves for honeycombs with $R=0$ and $R=0.6$ using re-calibrated material properties.

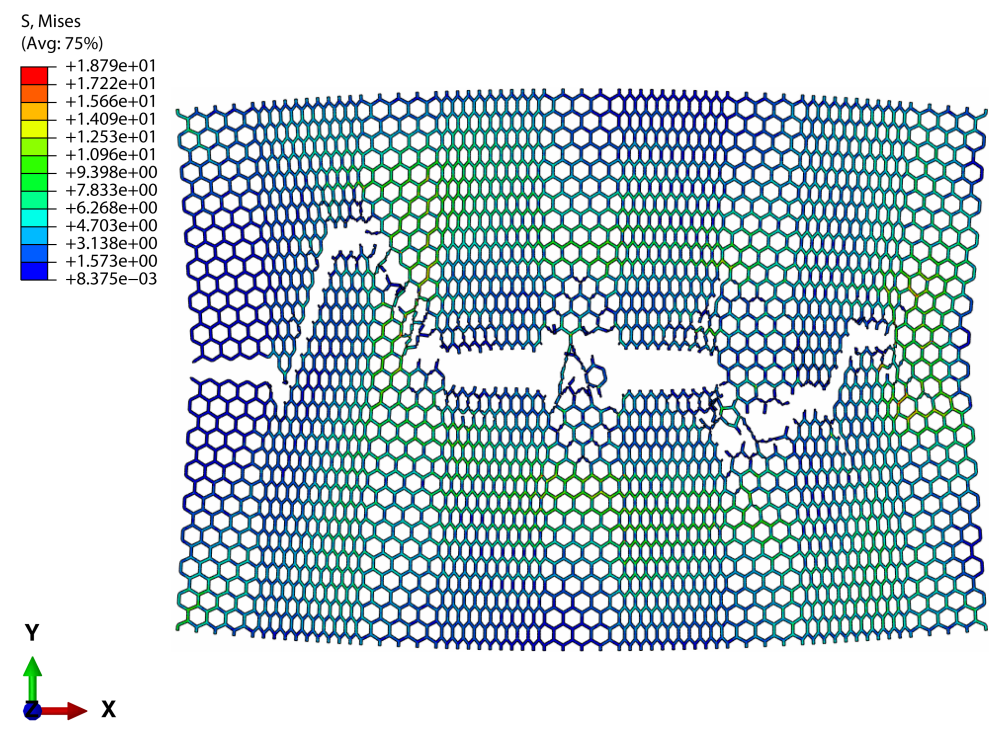

FIGURE 8: Crack path for a virtual specimen with $R=0.6$.

\section{REFERENCES}

1. N. A. Fleck, V. S. Deshpande, and M. F. Ashby, "Micro-architectured materials: past, present and future," Proceedings of the Royal Society A: Mathematical, Physical and Engineering Sciences 466, 2495-2516 (2010) https://royalsocietypublishing.org/doi/pdf/10.1098/rspa.2010.0215

2. J. Banhart, "Manufacture, characterisation and application of cellular metals and metal foams," Progress in Materials Science 46, 559 - 632 (2001)

3. M. Rashed, M. Ashraf, R. Mines, and P. J. Hazell, "Metallic microlattice materials: A current state of the art on manufacturing, mechanical properties and applications," Materials \& Design 95, 518 - 533 (2016)

4. Forbes, "Boeing creates the world's lightest metal," (2015).

5. U. Ramamurty and A. Paul, "Variability in mechanical properties of a metal foam," Acta Materialia 52, 869-876 (2004) 
6. B. Smith, S. Szyniszewski, J. Hajjar, B. Schafer, and S. Arwade, "Steel foam for structures: A review of applications, manufacturing and material properties," Journal of Constructional Steel Research 71, 1 - 10 (2012)

7. T. Frenzel, M. Kadic, and M. Wegener, "Three-dimensional mechanical metamaterials with a twist," Science 358, 1072-1074 (2017) https://science.sciencemag.org/content/358/6366/1072.full.pdf

8. J. Robbins, S. Owen, B. Clark, and T. Voth, "An efficient and scalable approach for generating topologically optimized cellular structures for additive manufacturing," Additive Manufacturing 12, 296 - 304 (2016) special Issue on Modeling \& Simulation for Additive Manufacturing.

9. Y. Brechet and J. Embury, "Architectured materials: Expanding materials space," Scripta Materialia 68, 1-3 (2013) architectured Materials.

10. M. A. Meyers and P.-Y. Chen, Biological materials science: biological materials, bioinspired materials, and biomaterials (Cambridge University Press, 2014).

11. R. Manno, W. Gao, and I. Benedetti, "A computational study on crack propagation in bio-inspired lattices," in Advances in Fracture and Damage Mechanics XVII Key Engineering Materials, Vol. 774 (Trans Tech Publications Ltd, 2018) pp. 398-404.

12. R. Manno, W. Gao, and I. Benedetti, "Engineering the crack path in lattice cellular materials through bio-inspired micro-structural alterations," Extreme Mechanics Letters 26, 8 - 17 (2019)

13. H. Ramezani Dana, F. Barbe, L. Delbreilh, A. Guillet, M. Ben Azzouna, and T. Breteau, "Polymer additive manufacturing of ABS Structure: influence of the deposition path on the tensile properties," Journal of Manufacturing Processes 44, 288-298 (2019).

14. C. Sohrabi, Z. Alsafi, N. O’Neill, M. Khan, A. Kerwan, A. Al-Jabir, C. Iosifidis, and R. Agha, "World health organization declares global emergency: A review of the 2019 novel coronavirus (covid-19)," International Journal of Surgery (2020). 